\title{
New Formulation Strategies in Topical Antifungal Therapy
}

\author{
Sevgi Güngör ${ }^{1 *}$, M. Sedef Erdal ${ }^{1}$, Buket Aksu $^{2}$ \\ ${ }^{1}$ Department of Pharmaceutical Technology, Faculty of Pharmacy, Istanbul University, Istanbul, Turkey; ${ }^{2}$ Santa Farma Drug Com- \\ pany, Istanbul, Turkey. \\ Email: *sgungor@istanbul.edu.tr
}

Received November $16^{\text {th }}, 2012$; revised December $14^{\text {th }}, 2012$; accepted December $23^{\text {rd }}, 2012$

\begin{abstract}
Fungal infections of the skin are one of the often faced with dermatological diseases in worldwide. Topical therapy is an attractive choice for the treatment of the cutaneous infections due to its advantageous such as targeting of drugs to the site of infection and reduction of the risk of systemic side effects. Currently, antifungal drugs are generally used as conventional cream and gel preparations in topical treatment. The efficiency of that treatment depends on the penetration of drugs through the target layers of the skin at the effective concentrations. However, stratum corneum, the outermost layer of the skin, is an effective barrier for penetration of drugs into deeper layers of the skin. The physicochemical characteristics of drug molecules and the types of the formulations are effective factors in topical drug delivery. Therefore, a number of formulation strategies have been investigated for delivering antifungal compounds through targeted site of the skin. This review article focuses on the new alternative formulation approaches to improve skin penetration of antifungal drugs.
\end{abstract}

Keywords: Antifungals; Colloidal Carriers; Vesicular Carriers; Particulate Carriers; Skin Delivery

\section{Introduction}

The incidence of superficial fungal infections of skin, hair and nails has been increased in worldwide. It has been estimated that about 40 million people have suffered from fungal infections in developing and under developed nations. The progression of fungal infections can be rapid and serious due to compromising with immune function [1,2]. Dermatophytes are one of the most frequent causes of tinea and onchomycosis. Candidal infections are also among the most widespread superficial cutaneous fungal infections [3]. Even, candida can invade deeper tissues as well as the blood which leads to lifethreating systemic candidiasis, when the immune system is weakened [4].

Topical treatment of fungal infections has several superiorities including, targeting the site of infection, reduction of the risk of systemic side effects, enhancement of the efficacy of treatment and, high patient compliance. Different type of topical effective antifungal compounds has been used in the treatment of a variety of dermatological skin infections. The main classes of topical antifungals are polyenes, azoles, and allylamine/benzylamines. Cicloprox is an antifungal agent also used topically. Currently, these antifungal drugs are commercially available in conventional dosage forms such as creams,

${ }^{*}$ Corresponding author. gels, lotions and sprays.

The efficiency of the topical antifungal treatment depends on the penetration of drugs through the target tissue. Hence, the effective drug concentration levels should be achieved in the skin. In topical administration of antifungals, the drug substances should pass the stratum corneum, which is the outermost layer of the skin, to reach lower layers of the skin, particularly into viable epidermis. In this context, the formulation may play a major role for penetration of drugs into skin [5]. Development of alternative approaches for topical treatment of fungal infections of skin encompasses new carrier systems for approved and investigational compounds. Delivery of antifungal compounds into skin can be enhanced with the carriers including colloidal systems, vesicular carriers, and nanoparticles.

This review article focuses on the classification of topical antifungals used in treatment of various superficial fungal infections of skin. Recent studies which deal with the optimization of alternative formulation approaches for cutaneous administration of antifungals have also been summarized.

\section{Topical Delivery of Antifungals via Skin}

Human skin is a well-organized membrane and, it has three main layers, which are called as epidermis, dermis and hypodermis. Stratum corneum, the outermost layer of 
epidermis is formed by dead and keratinized cells, and it is an excellent barrier to penetration of drugs through the skin [6].

Drugs should penetrate into skin layers to ensure effective drug concentrations following topical administration. Types of the formulations as well as the physicochemical characteristics of drug molecules are effective parameters in topical delivery of drugs. In topical administration, the entering of drugs to systemic circulation is prevented or minimized. Thus, the systemic adverse effects of drugs are avoided [7]. Besides, topical preparations have better patient compliance due to their noninvasiveness and, they can be self-administered $[8,9]$.

Antifungal drugs should reach effective therapeutic levels in viable epidermis after dermal administration. The greatest challenge for dermal delivery is stratum corneum, and in order to improve its permeability, new formulation approaches have been investigated. Colloidal drug carriers such as microemulsions, vesicular carriers including liposomes, ethosomes and niosomes and, both lipidic and polymeric particulate carrier systems are among those new carriers to ensure dermal administration of antifungals by dermal targeting $[10,11]$.

\section{The Conventional Dosage Forms and Action Mechanisms of Topical Antifungal Drugs Used in the Treatment of Dermal Fungal Diseases}

A list of approved and investigational topical antifungal compounds is given as a comprehensive table (Table 1). Topical antifungal agents are conventionally compounded into various types of vehicles, such as ointments, creams, lotions, gels, or sprays. In addition, several agents used perorally or intravenously are also included because of the conducted studies aiming dermal/transdermal targeting of these antifungals.

Azole antifungals work through a common mechanism of action; they selectively inhibit the synthesis of fungal cell ergosterol and they alter the permeability of cell membrane by binding with the phospholipids in the fungal cell membrane. The azole antifungal agents in clinical use contain either two or three nitrogens in the azole ring and are thereby classified as imidazoles (e.g., ketoconazole and miconazole, clotrimazole) or triazoles (e.g., itraconazole and fluconazole), respectively [3,12].

Allylamines work through inhibition of squalene epoxidase, which is an essential enzyme in the ergosterol biosynthesis pathway of fungal cell membrane formation. Alterations in fungal cellular membranes result in increased cellular permability and growth inhibition. Benzylamines block the epoxidation of squalene $[3,13]$.

The polyene antifungal agents exert their antifungal activity by binding irreversibly to fungal cell membrane ergosterol. Thus, the polyenes are fungicidal and have the broadest spectrum of antifungal activity of any of the clinically available agents. Nystatin is a polyene derivative and is limited to topical use only. Clinically, nystatin has demonstrated broad antifungal activity in treating mucocutaneous fungal infections. The most common adverse effect reported with nystatin is allergic contact dermatitis $[3,13,14]$.

Ciclopirox is a synthetic hydroxypyridone derivative that carries antifungal, antibacterial, and anti-inflammatory properties. Ciclopirox inhibits essential enzymes interfering with mitochondrial electron transport processes and energy production. It is active against many fungi including dermatophytes and yeast [3].

\section{Current Alternative New Formulation Approaches for Improving Treatment of Fungal Diseases in Skin}

\subsection{Colloidal Carriers (Microemulsions, Micelles, Nanoemulsions)}

Microemulsions, transparent, thermodinamically stable, and isotropic liquid dispersions, are promising colloidal carrier systems for topical and transdermal administration of drugs. The advantages of topical microemulsions include enhancing solubility of drugs, high thermodynamic stability, and ease of preparation and low costs [10]. The oils and surfactants included in the composition of microemulsions act as enhancers for permeation of drugs across stratum corneum. These formulation components of microemulsions and the internal structure of phases enhance diffusion of the drug and, that can improve the partition of drug to stratum corneum [54].

The optimisation and characterization of topical microemulsion formulations of antifungal drugs have been widely studied in the literature. Recently, El Hadidy et al. reported that microemulsion systems of voriconazole showed significantly better antifungal activity against Candida albicans than that of its supersaturated solution. Voriconazole permeation through pig skin has been prolonged up to $4 \mathrm{~h}$ with application of Jojoba oil-based microemulsion formulation [31].

In another study, Patel and co-workers has prepared oil in water $(\mathrm{o} / \mathrm{w})$ microemulsions of ketoconazole by using lauryl alcohol as oil, Labrasol as surfactant and ethanol as co-surfactant. It was concluded that the percutaneous absorption of ketoconazole from microemulsions has been enhanced with increasing the lauryl alcohol and water contents, and with decreasing the surfactant/ co-surfactant ratio in the formulation. In order to evaluate the antifungal activity of the best formulation, Candida albicans has been used as a model fungus. The results indicated that the widest zone of inhibition has been obtained with microemulsion formulation as compared to 


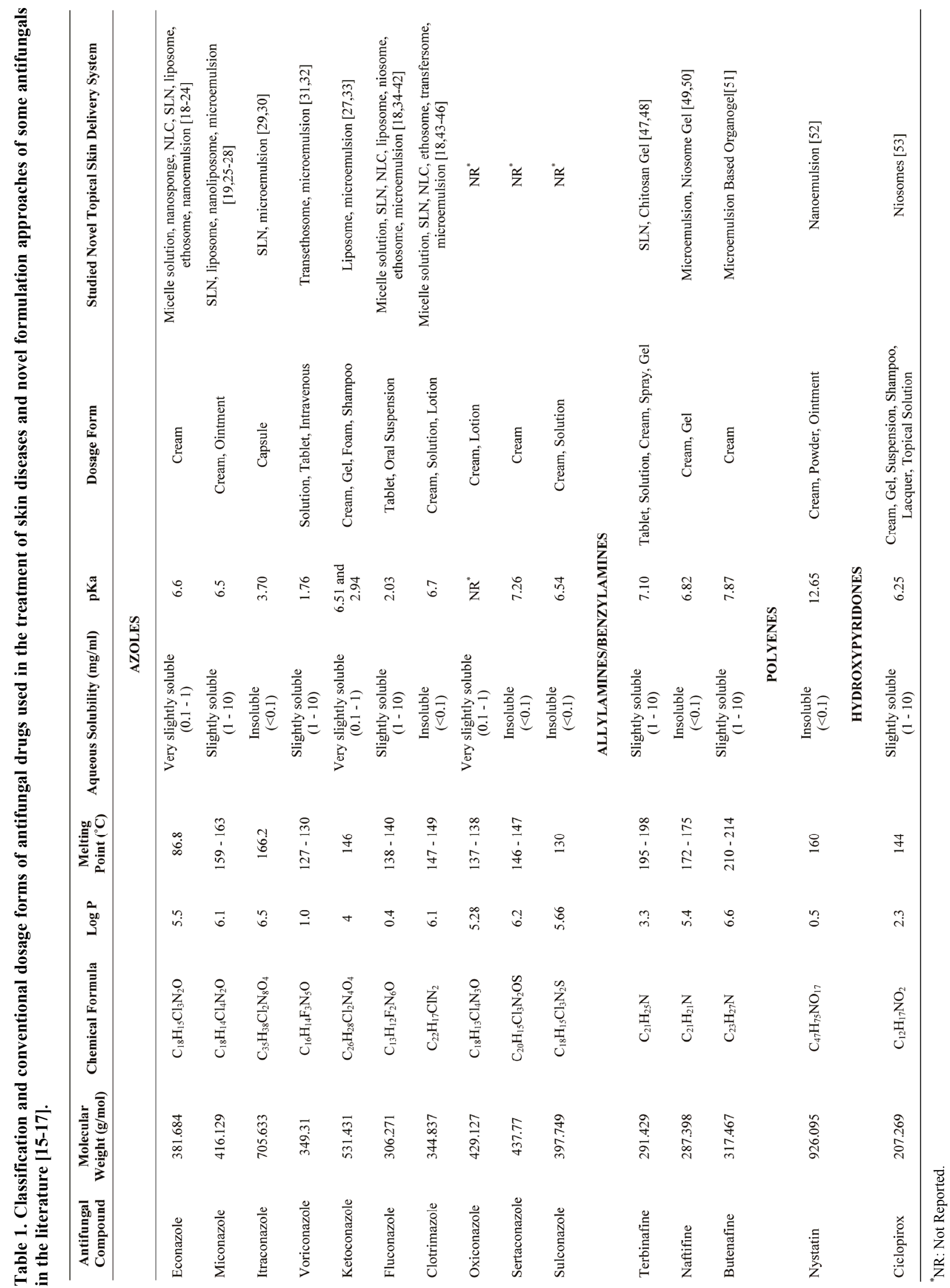


reference conventional formulation of ketaconazole. The safety of microemulsion formulations of ketoconazole for topical use has also been showed according to histopathological data on rat skin [33].

Microemulsion $(\mathrm{o} / \mathrm{w})$ of itraconazole was incorporated into polymeric gels prepared with Lutrol F127, Xanthan gum, or Carbopol 934. Diffusion controlled drug release mechanism has been followed from microemulsion based gels. When the antifungal activity of itraconazole was evaluated, the widest zone of inhibition has been observed with the application of microemulsion gels of itraconazole prepared with Lutrol F127. This microemulsion gels has been also identified as non-irritant [29].

Different type of topical delivery systems of fluconazole, including emulsion, emulgel, lipogel, and thickened microemulsion-based hydrogel have been evaluated to optimize the formulation type based on its drug delivery capacity to the skin and deposition of active compound within the skin. Propylene glycol and diethylene glycol monoethyl ether have been used as co-solvent and also as penetration enhancers for each system. Microemulsion formulation of fluconazole containing diethylene glycol monoethyl ether has been determined as the best formulation due to its ability to deliver the all applied dose into skin and to enhance skin penetration of fluconazole. Also, it has been reported that microemulsion formulation prepared with diethyleneglycolmonoethyl ether had higher antifungal activity compared to the one containing propylene glycol [34].

In another study, fluconazole loaded microemulsion systems composed of lauryl alcohol (oil), Labrasol (surfactant) and ethanol (co-surfactant) have been formulated and the effect of formulation components on in vitro skin permeation of fluconazole has been investigated. It was observed that the percutaneous absorption of fluconazole from microemulsions has been enhanced with increasing the oil and water contents, and with decreasing the surfactant/co-surfactant ratio in the formulation. The antifungal activity of the best microemulsion formulation has been assessed in terms of the zone of inhibition of Candida albicans, data indicated the widest zone of inhibition has been shown with the application of the selected microemulsion formulation [35].

Microemulsion based lecithin organogel formulations for topical delivery of fluconazole has been developed and their suitability has been investigated in order to overcome the gastrointestinal adverse effects of the drug. The transdermal permeability of fluconazole from different concentrations of lecithin organogels has been examined through excised rat skin. Organogel containing lecithin has showed the higher drug release and better relative consistency and that formulation has also showed an increase in antifungal activity [36].

The influence of the vehicle on the release and per- meation of fluconazole dissolved in Jojoba oil was evaluated by El Laithy and El-Shaboury. Series of Cutinalipogels (Cutina CPA [cetylpalmitate], CBS [mixture of glyceryl stearate, cetearyl alcohol, cetylpalmitate, and cocoglycerides], MD [glyceryl stearate], and GMS [glycerylmonostearate]) in different concentrations as well as gel microemulsion were prepared. Candida albicans was used as a model fungus to evaluate the antifungal activity of the best formula achieved. The highest values were assured from gel microemulsion formulation and the antifungal activity of fluconazole showed the widest zone of inhibition with gel microemulsion [37].

Microemulsion systems for topical delivery of clotrimazole were developed by using either lemon oil or isopropylmyristate as the oil phase and Tween 80 and nbutanol as surfactant and co-surfactant, respectively. Gel forms of microemulsions were prepared using Carbopol 934 as the hydrogel matrix. The microemulsion gel system comprising of isopropyl myristate, Tween 80 , nbutanol and water was determined as a successful topical delivery system of clotrimazole for treatment of cutaneous fungal infections because of the efficacy, tolerability and high skin retention of the drug [43].

Zhao et al., developed gelatine containing microemulsion-based organogels loaded with butenafine hydrochloride as a model drug. They observed that the network structure of microemulsion gels composed of isopropyl myristate, sodiumbis(2-ethyl-1-hexyl) sulfosuccinate, Tween 85 and water was affected by drug concentration and the results showed that the microemulsion gels can be used as potentially transdermal drug delivery vehicles [51].

Micelles are nanosized colloidal carries with a hydrophobic core and hydrophilic shell. They are used as pharmaceutical carriers for water insoluble drugs and are attractive drug carriers providing increased bioavailability [55]. The specificity and efficacy of micelle-based drug delivery can be improved through the use of $\mathrm{pH}-$, thermo-, ultrasound-, or light-sensitive block copolymers and the attachment of targeting ligands to the micelles [56]. Bachav et al., studied the effect of miceller carriers of different azole group of antifungal drugs including, clotrimazole, econazole nitrate and fluconazole to improve the skin deposition of antifungals following topical administration. In this study, aqueous micelle solutions of these compounds have been formulated using novel amphiphilicmethoxy-poly(ethylene glycol)-hexyl substituted polylactide (MPEG-hexPLA) block copolymers. In vitro skin transport studies performed with using full thickness porcine and human skin for 6 hours indicated that econazole deposition in porcine skin has been found significantly higher than that from its commercial liposomal formulation. The increase in econazole skin deposition achieved using the micelle formulation has been 
explained by the ability of micelle formulation to improve cutaneous drug bioavailability [18].

Submicron emulsions or nanoemulsions also have been evaluated as colloidal carriers to improve the efficacy and tolerability of antifungal drugs applied topically. Nanoemulsions are thermodynamically stable, transparent or translucent nano-sized dispersions of oil in water $(\mathrm{o} / \mathrm{w})$ or water in oil (w/o) stabilized by an interfacial film of surfactant and co-surfactant.

They are primarily produced either by high-energy emulsification (e.g., high-pressure homogenization) or by low-energy emulsification (using physicochemical properties of the components) $[57,58]$.

A nystatine nanoemulsion for topical application was developed to avoid undesirable side effects as systemic absorption and toxicity. Ex vivo human skin permeation studies showed that the retained amount of drug was sufficient to ensure an antifungal effect and nystatin was not absorbed into systemic circulation [52].

Econazole or miconazole nitrate, respectively, were incorporated in positively and negatively charged submicron emulsions. It was observed that there was a relationship between the charge of the emulsion and skin permeation (Piemi et al., 1999). In another study, skin permeation and accumulation of miconazole nitrate from positively charged microemulsions were investigated. The accumulation of miconazole nitrate from positively charged microemulsions was nearly twice that from their negatively charged counterparts without a concomitant increase in its systemic absorption [25].

Microemulsion formulations of naftifine hydrochloride have been optimized and the efficacy of the formulation has been investigated by ATR-FTIR spectroscopy. According to ATR-FTIR data, microemulsion carrier systems have enhanced the permeation of naftifine hydrochloride, highly lipophilic drug, through stratum corneum, indicating the localization of antifungal compound in deeper layers of the skin [49].

\subsection{Vesicular Carriers (Liposomes, Ethosomes, Niosomes and Transfersomes)}

Vesicular systems such as liposomes, niosomes, ethosomes and transfersomes have been developed for optimization of penetration of drugs across stratum corneum and particularly for dermal targeting of the drug compounds. Vesicular systems have numerous advantages such as controlling drug release rate from the applied formulation and localization of topical administered drugs in targeted dermal layers. Besides, transdermal administration of vesicular systems helps to carry drug molecules into systemic circulation.

Liposomes are defined as lipidic vesicles containing water. Conventional liposomes are composed of phospholipids, mostly phosphatidylcholine from soybean or egg yolk, with or without cholesterol [59]. Liposomes are accepted as ideal dermal drug carriers due to their ability to alter the biodistribution profile of incorporated drugs [38]. Liposomes can be adsorbed on the skin surface or may go into fusion. Fusion of liposomes may facilitate dermal penetration of the drug. Another skin penetration enhancement mechanism is penetration of liposomes to stratum corneum to some extent before fusion with stratum corneum lipids, which leads to deposition of the drug in tissue. With this mechanism, particularly dermally administered liposomes can be localized in different layers of the skin $[6,60]$.

Schwarz and co-workers employed lysine derivatives to optimize the liposomal microstructure. The effect of the oligopeptides Lys-5 and Lys-7 on the structure as well as on the skin permeation of the antimycotic drug fluconazole in

1,2-dipalmitoyl-sn-glycero-3-phosphocholine vesicles was studied using a variety of techniques. The addition of Lys5 and Lys-7 induced a structural change resulting in a decrease in particle size between $10 \%$ and $40 \%$ in liposomes consisted mainly of unilamellar vesicles and a retarding effect on fluconazole skin permeation [38].

The antifungal activity of liposomes loaded with miconazole has been assessed using Candida albicans, and in vitro skin penetration of liposome formulations of miconazole have been also examined across human skin. The results showed that propylene glycol-phospholipid vesicles provided enhanced skin deposition of miconazole nitrate with minimum skin permeation [26]. In another study, the incorporation of miconazole or ketoconazole in liposomes was resulted in a delay and decreased antifungal activity of the drugs due to the various phosholipid concentrations and different liposomal preparations [27].

The effects of two commercially available econazole formulations (econazole nitrate cream, econazole liposome gel) on both uninfected reconstructed human epidermis and on a model of human cutaneous candidosis have been investigated with the light and electron microscopy. The data obtained indicated that barrier damage on epidermis and irritativetoxic effects caused by a single application of the cream to the uninfected reconstructed epidermis were more than the liposome gel. It was also shown that different Candida albicans-specific alterations, namely hyperkeratosis, focal thickening of the stratumcorneum, dyskeratosis and parakeratosis have been totally eliminated following the application of the liposome formulation [20].

Niosomes are a kind of liposomes prepared with nonionic surfactants. Niosomes have advantages in terms of cost, and chemical stability compared to conventional liposomes [59]. Dermal penetration of niosomes depends on potential penetration-enhancing activity of surfactants 
in its content, penetration of the vesicle to stratum corneum, accumulation of vesicle on the skin surface and/or increasing thermodynamic activity of the drug on the skin surface. These mechanisms depend on the physicochemical properties of the drug, the vesicle and the lipids used $[6,61]$. In the first studies, niosomes mostly investigated to encapsulate cosmetic compounds, but then they have been also evaluated as drug delivery carriers. Numerious studies also reported on the formulation optimisation of niosomes loaded with antifungal compounds in the literature.

Fluconazole loaded liposomes and niosomes were prepared by the lipid/non-ionic surfactant-based dry-film hydration method and incorporated into Carbopol gel $(1 \% ; \mathrm{w} / \mathrm{w})$ for sustained, localized application to achieve a localized therapeutic concentration of drug in skin. The skin-retention studies of fluconazole from in vitro and in vivo experiments showed significantly higher accumulation of drug in the case of liposomal or niosomal gels compared with plain gel (14.2 and 3.3-fold higher, respectively) [39].

Fluconazole-loaded niosomes composed of Span 40, Span 60, and Brij 72 have been formulated by the film hydration method. In vitro skin permeation and retention studies showed that niosomes form localized drug depots in the skin, indicating sustained drug release and enhancement cutaneous retention of fluconazole [40].

In another study, an alcohol-free niosome gel containing naftifine hydrochloride has been developed and formulation has been optimized to achieve maximum entrapment coupled with stability. Negatively charged niosomes has also been incorporated into a hydroxyethylcellulose gel [50].

Niosomes also has been evaluated as carriers for dermal delivery of ciclopiroxolamine. The results suggested that niosomes consisting of Span 60, cholesterol and diacetyl phosphate are promising delivery systems for cutaneous retention of ciclopirox [53].

Ethosomes contain phospholipids like classical liposomes; however, they also contain high levels of alcohol. It has been demonstrated that its components can reach deeper layers of the skin or enter into systemic circulation [62]. High ethanol content of ethosomes can enhance the solubility of more lipophilic drugs (Morrow et al., 2007). Action mechanism of these carriers in improving permeation has been explained by presence of alcohol as penetration enhancers. Ethanol disrupt intercellular lipid structure of stratum corneum by the phospholipids in their content $[6,63]$.

Ethanolic nanovesicles of econazole nitrate have been optimized and compared with liposomal and hydro-ethanolic gels. Ethosomal gel of econazole nitrate has been found to have outstanding potential to serve as a topical delivery system, having controlled drug release, provid- ing better antifungal activity, and good storage stability [4].

Clinical efficacy of fluconazole encapsulated ethosomes in a suitable dermatological base for the treatment of candidiasis patients has been assessed against liposomal gel, marketed product and hydro-ethanolic solution of the drug. From the clinical evaluation, the developed novel delivery system demonstrated enhanced antifungal activity compared to liposomal formulation, marketed formulation and hydroethanolic solution of the drug [41].

Song et al. showed that transethosomes has enhanced significantly the skin permeation of voriconazole compared to the control and other vesicles such as deformable liposomes, conventional liposomes. They also indicated that in vitro and in vivo skin deposition of voriconazole in the dermis/epidermis region has been improved with the application of ethosomal formulations of voriconazole [32].

Transfersomes are called as highly deformable, or elastic liposomes. They are composed of phospholipids and a surfactant which gives flexibility to the liposome structure. Transfersomes have been successfully assesed as topical and transdermal carriers for drugs and have also been shown to be effective carriers for genetic material and vaccines [59].

Transfersomes have been evaluated as carrier for topical antifungal administration. Clotrimazole loaded ethosomes have led to fluidization and extraction of skin lipids after treatment, which was resulted in greater drug flux compare to that of transfersomes (ultradeformable liposomes) [44].

\subsection{Nanoparticulate Carriers}

The nanoparticulate carrier systems as solid lipid nanoparticles (SLNs) and nanostructured lipid carriers (NLCs) have gained interest for the topical treatment of skin associated fungal infection as they facilitate the skin penetration of loaded drugs [42].

SLN are water-in-oil emulsions containing solids as oil phase, and these systems are prepared from solid lipids or from blends of the lipids. NLCs are defined as new generation of lipid particles, which have been developed to overcome certain limitations of SLNs. NLCs contain mixtures of different solid lipids blended with liquid oils. The most important advantage of these carriers is to have low risk of toxicity. Small size of lipid particles ensures close contact with stratum corneum, and may enhance dermal penetration of drug [45]. These carriers offer controlled release profiles for many compounds [42]. In recent years SLN and NLC carries have been evaluated as carrier for antifungal compounds.

Recently, fluconazole loaded SLNs and NLCs were prepared and characterized for different parameters. The 
data obtained from in vitro and in vivo experiments revealed that significantly higher amount of drug accumulation was observed in skin with the application of NLCs formulation. The antifungal efficacy study has been performed on experimentally induced cutaneous Candidiasis in immunosuppressed albino rats, the findings confirmed the maximum therapeutic efficacy of NLCs. It was concluded that NLCs provided a good skin targeting effect and might be a promising carrier for topical delivery of fluconazole offering the sustained release and maintain the localized effect, resulting in an effective treatment of a life-threatening cutaneous fungal infection [42]. In another study, topical NLC delivery system of econazole nitrate for the treatment of deep seated fungal infection has been developed to improve drug permeability [21].

SLNs formulations of terbinafine have been formulated to overcome the problems of long treatment durations and frequent administration. In vitro penetration levels of terbinafine of the designed formulations and a commercial product, in the stratum corneum, viable epidermis, and dermis were measured. It was observed that the amount of terbinafine penetrated the skin layers have been enhanced by increasing the percentage of the lipid phase of the formulation. The authors also concluded that the application of terbinafine with SLNs formulations could resolve the practical problem of the longer administration period [47].

Sanna et al. showed that SLN formulations have promoted a rapid penetration of econazol nitrate across stratum corneum after $1 \mathrm{~h}$ and have improved the penetration of the drug into deeper skin layers after $3 \mathrm{~h}$ of application compared to reference gel [22]. Passerini et al. have also compared econazole nitrate loaded SLNs to solid lipid microparticles having identical formulation components. The results indicated that econazole delivery kinetics in porcine skin was not size dependent [23].

It was observed that SLN dispersions of miconazole nitrate significantly have increased the accumulative uptake of miconazole nitrate in skin over the commercial gel preparation and SLN dispersions also showed a significantly enhanced skin targeting effect [28].

Mukherjee and co-workers designed and evaluated itraconazole loaded solid lipid nanoparticles SLNs to improve the therapeutic efficacy and reduction of the antifungal agent. The in vitro drug release profile from SLNs have prolonged up to 12 hours [30].

Clotrimazole-loaded SLNs and NLCs have led to modified drug release over a period of 10 hours [45]. In another study, it was also shown that both SLN and NLC formulations loaded with clotrimazole had a sustained/ prolonged drug release [46].

\subsection{Gelling Systems-Polymeric Carriers}

The feasibility of econazole nitrate loaded polymeric nanosponges has been evaluated. The nanosponges containing polyvinyl alcohol:ethyl cellulose $(3: 2)$ have been formulated with Carbopol $934 \mathrm{NF}$ as hydrogel using varying concentrations of permeation enhancers such as propylene glycol and N-methyl-2-pyrrolidone. The optimization studies showed that econazole nitrate was stable in the nanosponge delivery system and there was no drugpolymer interaction in nanosponges [24].

Topical hydrogel formulations of terbinafine hydrochloride have been optimized with using different types of chitosan at different molecular weight. The antifungal inhibitory activity of the formulation has been evaluated. The results indicated that a higher drug release and the highest zone of inhibition were obtained from hydrogels prepared with the lowest molecular weight chitosan compared to that of other chitosan gels and marketed commercial product [48].

\section{Conclusion}

Topical treatment of the cutaneous infections has been preferred due to its superiorities over oral treatment such as avoidance of systemic adverse effects, targeting of the drug on the site of infection, and high patient compliance. On the other hand, adequate drug concentrations in target layers of the skin should be provided to ensure the efficacy of topical treatment. Thus, delivery of antifungals to target region of the skin is a great challenge in terms of therapeutic aspect. In this context, formulation of topical product plays a key role for penetration of the drugs across skin. Besides, the physicochemical properties of drug molecules such as lipophilicity are also effective parameter. Generally, antifungal drugs are highly lipophilic compounds, which can affect the penetration of drugs across stratum corneum. Various formulation strategies have emerged over recent years to optimize new drug delivery carriers of antifungal drugs, and some promissing data, to some extent, have been published.

\section{REFERENCES}

[1] M. Ameen, "Epidemiology of Superficial Fungal Infections," Clinical Dermatology, Vol. 28, No. 2, 2010, pp. 197201. doi:10.1016/j.clindermatol.2009.12.005

[2] B. Havlickova and M. Friedrich, "Epidemiological Trends in Skin Mycoses Worldwide," Mycoses, Vol. 51, Suppl. 4, 2008, pp. 2-15. doi:10.1111/j.1439-0507.2008.01606.x

[3] A. Y. Zhang, W. L. Camp and B. E. Elewski, "Advances in Topical and Systemic Antifungals," Clinical Dermatology, Vol. 25, No. 2, 2007, pp. 165-183. doi:10.1016/j.det.2007.01.002

[4] P. Vermaand and K. Pathak, "Nanosized Ethanolic Vesicles Loaded with Econazole Nitrate for the Treatment of Deep Fungal Infections through Topical Gel Formulation," Nanomedicine, Vol. 8, No. 4, 2012, pp. 489-496. doi:10.1016/j.nano.2011.07.004 
[5] C. M. Lee and H. I. Maibach, "Deep Percutaneous Penetration into Muscles and Joints," Journal of Pharmaceutical Science, Vol. 95, No. 7, 2006, pp. 1405-1412. doi:10.1002/jps.20666

[6] A. Williams, "Transdermal and Topical Drug Delivery: From Theory to Clinical Practice," Pharmaceutical Press, London, 2003.

[7] R. H. Guy, "Current Status and Future Prospects of Transdermal Drug Delivery," Pharmaceutical Research, Vol. 13, No. 12, 1996, pp. 1765-1769. doi:10.1023/A:1016060403438

[8] R. H. Guy, “Transdermal Drug Delivery,” In: M. SchäferKorting, Ed., Drug Delivery, Handbook of Experimental Pharmacology, Springer-Verlag, Berlin, 2010.

[9] T. Taner and R. Mark, "Delivering Drugs by the Transdermal Route: Review and Comment," Skin Research Technology, Vol. 14, No. 3, 2008, pp. 249-260. doi:10.1111/j.1600-0846.2008.00316.x

[10] H. R. Neubert, "Potentials of New Nanocarriers for Dermal and Transdermal Drug Delivery," European Journal of Pharmacy Biopharmacy, Vol. 77, No. 1, 2011, pp. 1-2. doi:10.1016/j.ejpb.2010.11.003

[11] H. A. Benson, "Elastic Liposomes for Topical and Transdermal Drug Delivery," Current Drug Delivery, Vol. 6, No. 3, 2009, pp. 217-226. doi:10.2174/156720109788680813

[12] D. J. Sheehan, C. A. Hitchcock and C. M. Sibley, "Current and Emerging Azole Antifungal Agents," Clinical Microbiology Reviews, Vol. 12, No. 1, 1999, pp. 40-79.

[13] V. T. Andriole, "Current and Future Antifungal Therapy: New Targets for Antifungal Therapy," International Journal of Antimicrobial Agents, Vol. 44, No. 2, 1999, pp. 151162.

[14] B. Di Domenico, "Novel Antifungal Drugs," Current Opinion in Microbiology, Vol. 2, No. 5, 1999, pp. 509-515. doi:10.1016/S1369-5274(99)00009-0

[15] M .J. O’Neil, “The Merck Index: An Encyclopedia of Chemicals, Drugs and Biologicals," 14th Edition, Merck \& Co Inc., Rahway, 2006.

[16] ChemBlink, "Online Database of Chemicals from Around the World," 2012. http://www.chemblink.com/products

[17] Drug Bank, "Open Data Drug and DrugTarget Database," 2012. http://www.drugbank.ca/

[18] Y. G. Bachhav, K. Mondon, Y. N. Kalia, R. Gurny and M. Möller, "Novel Micelle Formulations to Increase Cutaneous Bioavailability of Azole Antifungals," Journal of Controlled Release, Vol. 153, No. 2, 2011 pp. 126-132. doi:10.1016/j.jconrel.2011.03.003

[19] M. P. Piemi, D. Korner, S. Benita and J. P. Marty, "Positively and Negatively Charged Submicron Emulsions for Enhanced Topical Delivery of Antifungal Drugs," Journal of Controlled Release, Vol. 58, No. 2, 1999, pp. 177187. doi:10.1016/S0168-3659(98)00156-4

[20] M. Schaller, H. Preidel, E. Januschke and H. C. Korting, "Light and Electron Microscopic Findings in a Model of Human Cutaneous Candidosis Based on Reconstructed Human Epidermis Following the Topical Application of Different Econazole Formulations," Journal of Drug Tar- geting, Vol. 6, No. 5, 1999, pp. 361-372. doi: $10.3109 / 10611869908996843$

[21] L. Keshriand and K. Pathak, "Development of Thermodynamically Stable Nanostructured Lipid Carrier System Using Central Composite Design for Zero Order Permeation of Econazole Nitrate through Epidermis," Pharmaceutical Development and Technology, 2012, Epub ahead of Print.

[22] V. Sanna, E. Gavini, M. Cossu, G. Rassu and P. Giunchedi, "Solid Lipid Nanoparticles (SLN) as Carriers for the Topical Delivery of Econazole Nitrate: In-Vitro Characterization, ex-Vivo and in-Vivo Studies," Journal of Pharmacy and Pharmacology, Vol. 59, No. 8, 2007, pp. 1057 1064. doi:10.1211/jpp.59.8.0002

[23] N. Passerini, E. Gavini, B. Albertini, G. Rassu, M. Di Sabatino, V. Sanna, P. Giunchedi and L. Rodriguez, "Evaluation of Solid Lipid Microparticles Produced by Spray Congealing for Topical Application of Econazole Nitrate," Journal of Pharmacy and Pharmacology, Vol. 61, No. 5, 2009, pp. 559-567. doi:10.1211/jpp.61.05.0003

[24] R. Sharma and K. Pathak, "Polymeric Nanosponges as an Alternative Carrier for Improved Retention of Econazole Nitrate onto the Skin through Topical Hydrogel Formulation," Pharmaceutical Development and Technology, Vol. 16, No. 4, 2011, pp. 367-376. doi:10.3109/10837451003739289

[25] E. Peira, M. E. Carlotti, C. Trotta, R. Cavalli and M. Trotta, "Positively Charged Microemulsions for Topical Application," International Journal of Pharmacy, Vol. 346, No. 1-2, 2008, pp. 119-123. doi:10.1016/j.ijpharm.2007.05.065

[26] R. M. Elmoslemany, O. Y. Abdallah, L. K. El-Khordagui and N. M. Khalafallah, "Propylene Glycol Liposomes as a Topical Delivery System for Miconazole Nitrate: Comparison with Conventional Liposomes," AAPS Pharmaceutical Science Technology, Vol. 13, No. 2, 2012, pp. 723731. doi:10.1208/s12249-012-9783-6

[27] A. D. Logu, A. M. Fadda, C. Anchisi, A. M. Maccioni, C. Sinico, M. L. Schivo and F. Alhaique, "Effects of in-Vitro Activity of Miconazole and Ketoconazole in Phospholipid Formulations," Journal of Antimicrobial Chemotherapy, Vol. 40, No. 6, 1997, pp. 889-893. doi:10.1093/jac/40.6.889

[28] M. R. Bhalekar, V. Pokharkar, A. Madgulkar and N. Patil, "Preparation and Evaluation of Miconazole Nitrate-Loaded Solid Lipid Nanoparticles for Topical Delivery," AAPS Pharmaceutical Science Technology, Vol. 10, No. 1, 2009, pp. 289-296. doi:10.1208/s12249-009-9199-0

[29] A. Chudasama, V. Patel, M. Nivsarkar, K. Vasu and C. Shishoo, "Investigation of Microemulsion System for Transdermal Delivery of Itraconazole," Journal of Advanced Pharmaceuical Technology Research, Vol. 2, No. 1, 2011, pp. 30-38.

[30] S. Mukherjee, S. Ray and R. S. Thakur, "Design and Evaluation of Itraconazole Loaded Solid Lipid Nanoparticulate System for Improving the Antifungal Therapy," Pakistan Journal of Pharmaceutical Science, Vol. 22, No. 2, 2009, pp. 131-138.

[31] G. N. El-Hadidy, H. K. Ibrahim, M. I. Mohamed and M. 
F. El-Milligi, "Microemulsions as Vehicles for Topical Administration of Voriconazole: Formulation and in Vitro Evaluation," Drug Development and Industrial Pharmacy, Vol. 38, No. 1, 2012, pp. 64-72. doi:10.3109/03639045.2011.590731

[32] C. K. Song, P. Balakrishnan, C. K. Shim, S. J. Chung, S. Chong and D. D. Kim, "A Novel Vesicular Carrier, Transethosome, for Enhanced Skin Delivery of Voriconazole: Characterization and in Vitro/in Vivo Evaluation," Colloids and Surfaces: B Biointerfaces, Vol. 92, 2012, pp. 299304. doi:10.1016/j.colsurfb.2011.12.004

[33] M. R. Patel, R. B. Patel, J. R. Parikh, A. B. Solanki and B. G. Patel, "Investigating Effect of Microemulsion Components: In vitro Permeation of Ketoconazole," Pharmaceutical Development and Technology, Vol. 16, No. 3, 2011, pp. 250-258. doi:10.3109/10837451003610845

[34] C. Salerno, A. M. Carlucci and C. Bregni, "Study of in Vitro Drug Release and Percutaneous Absorption of Fluconazole from Topical Dosage Forms," AAPS Pharmaceutical Science Technology, Vol. 11, No. 2, 2010, pp. 986993. doi:10.1208/s12249-010-9457-1

[35] M. R. Patel, R. B. Patel, J. R. Parikh, A. B. Solanki and B. G. Patel, "Effect of Formulation Components on the in Vitro Permeation of Microemulsion Drug Delivery System of Fuconazole," AAPS Pharmaceutical Science Technology, Vol. 10, No. 3, 2009, pp. 917-923. doi:10.1208/s12249-009-9286-2

[36] K. R. Jadhav, V. J. Kadam and S. S. Pisal, "Formulation and Evaluation of Lecithin Organogel for Topical Delivery of Fluconazole," Current Drug Delivery, Vol. 6, No.2, 2009, pp. 174-813. doi:10.2174/156720109787846252

[37] H. M. El Laithy and K. M. El-Shaboury, "The Development of Cutina Lipogels and Gel Microemulsion for Topical Administration of Fluconazole," AAPS Pharmaceutical Science Technology, Vol. 3, No. 4, 2002, p. E35.

[38] J. C. Schwarz, H. Kählig, N. B. Matsko, M. Kratzel, M. Husa and C. Valenta, "Decrease of Liposomal Size and Retarding Effect on Fluconazole Skin Permeation by Lysine Derivatives," Journal of Pharmaceutical Science, Vol. 100, No. 7, 2011, pp. 2911-2919. doi:10.1002/jps. 22513

[39] M. Gupta, A. K. Goyal, S. R. Paliwal, R. Paliwal, N. Mishra, B. Vaidya, D. Dube, S. K. Jain and S. P. Vyas, "Development and Characterization of Effective Topical Liposomal System for Localized Treatment of Cutaneous Candidiasis," Journal of Liposome Research, Vol. 20, No. 4, 2010, pp. 341-50. doi:10.3109/08982101003596125

[40] M. Gupta, B. Vaidya, N. Mishra and S. P. Vyas, "Effect of Surfactants on the Characteristics of Fluconazole Niosomes for Enhanced Cutaneous Delivery," Artificial Cells, Blood Substitutes, and Immobilization Biotechnology, Vol. 36, No. 6, 2011, pp. 376-834. doi:10.3109/10731199.2011.611476

[41] M. K. Bhalaria, S. Naik and A. N. Misra, "Ethosomes: A Novel Delivery System for Antifungal Drugs in the Treatment of Topical Fungal Diseases," Indian Journal of Experimental Biology, Vol. 47, No. 5, 2009, pp. 368-375.

[42] M. Gupta and S. P. Vyas, "Development, Characterization and in Vivo Assessment of Effective Lipidic Nano- particles for Dermal Delivery of Fluconazole against $\mathrm{Cu}$ taneous Candidiasis," Chemistry Physics of Lipids, Vol. 165, No. 4, 2012, pp. 454-461. doi:10.1016/i.chemphyslip.2012.01.006

[43] F. M. Hashem, S. D. Shaker, M. K. Ghorab, M. Nasr and A. Ismail, "Formulation, Characterization, and Clinical Evaluation of Microemulsion Containing Clotrimazole for Topical Delivery," AAPS Pharmaceutical Science Technology, Vol. 12, No. 3, 2011, pp. 879-886. doi:10.1016/j.chemphyslip.2012.01.006

[44] R. G. S. Maheshwari, R. K. Tekade, P. A. Sharma, G. Darwhekar, A. Tyagi, R. P. Patel and D. K. Jain, "Ethosomes and Ultradeformable Liposomes for Transdermal Delivery of Clotrimazole: A Comparative Assessment," Saudi Pharmaceutical Journal, Vol. 20, No. 2, 2012, pp. 161-170. doi:10.1016/j.chemphyslip.2012.01.006

[45] E. B. Souto, S. A. Wissing, C. M. Barbosa and R. H. Müller, "Development of a Controlled Release Formulation Based on SLN and NLC for Topical Clotrimazole Delivery," International Journal of Pharmacy, Vol. 278, No. 1, 2004, pp. 71-77. doi:10.1016/j.ijpharm.2004.02.032

[46] S. Das, W. K. Ng and R. B. Tan, "Are Nanostructured Lipid Carriers (NLCs) Better than Solid Lipid Nanoparticles (SLNs): Development, Characterizations and Comparative Evaluations of Clotrimazole-Loaded SLNs and NLCs," European Journal of Pharmaceutical Science, Vol. 47, No. 1, 2012, pp. 139-151. doi:10.1016/i.ejps.2012.05.010

[47] Y. C. Chen, D. Z. Liu, J. J. Liu, T. W. Chang, H. O. Ho and M. T. Sheu, "Development of Terbinafine Solid Lipid Nanoparticles as a Topical Delivery System," International Journal of Nanomedicine, Vol. 7, 2012, pp. 44094418.

[48] I. Ozcan, O. Abaci, A. H. Uztan, B. Aksu, H. Boyacioğlu, T. Güneri and O. Ozer, "Enhanced Topical Delivery of Terbinafine Hydrochloride with Chitosan Hydrogels," AAPS Pharmaceutical Science Technology, Vol. 10, No. 3, 2009, pp. 1024-1031. doi:10.1208/s12249-009-9299-X

[49] M. S. Erdal, S. Güngör and Y. Özsoy, "Microemulsions of Naftifine Hydrochloride: Characterization of Impact on Stratum Corneum Permeability with ATR-FTIR Spectroscopy," European Journal of Pharmaceutical Sciences, Vol. 44, 2011, pp. 159-160.

[50] H. S. Barakat, I. A. Darwish, L. K. El-Khordagui and N. M. Khalafallah, "Development of Naftifine Hydrochloride Alcohol-Free Niosome Gel," Drug Development and Industrial Pharmacy, Vol. 35, No. 5, 2009, pp. 631-637. doi:10.1080/03639040802498864

[51] X. Y. Zhao, Q. Cao, L. Q. Zheng and G. Y. Zhang, "Rheological Properties and Microstructures of Gelatin Containing Microemulsion-Based Organogels, Colloids and Surfaces A," Physicochemistry Engineering Aspects, Vol. 281, No. 1, 2006, pp. 67-73. doi:10.1016/j.colsurfa.2006.02.051

[52] F. Fernández-Campos, B. C. Naveros, O. L. Serrano, C. A. Merino and A. C. C. Campmany, "Evaluation of Novel Nystatin Nanoemulsion for Skin Candidosis Infections," Mycoses, Vol. 56, No. 1, 2013, pp. 70-81. doi:10.1111/j.1439-0507.2012.02202.x 
[53] K. S. Shaikh, B. Chellampillai and A. P. Pawar, "Studies on Nonionic Surfactant Bilayer Vesicles of Cicloprox Olamine," Drug Development and Industrial Pharmacy, Vol. 36, No. 8, 2010, pp. 946-953. doi: $10.3109 / 03639040903585150$

[54] A. Kogan and N. Garti, "Microemulsions as Transdermal Drug Delivery Vehicles," Advances in Colloid and Interface Science, Vol. 123-126, 2006, pp. 369-385. doi:10.1016/i.cis.2006.05.014

[55] V. P. Torchilin, "Micellar Nanocarriers: Pharmaceutical Perspectives," Pharmaceutical Research, Vol. 24, No. 1, 2006, pp. 1-16. doi:10.1007/s11095-006-9132-0

[56] C. Oerlemans, W. Bult, M. Bos, G. Storm, J. F.W. Nijsen, and W. E. Hennink, "Polymeric Micelles in Anticancer Therapy: Targeting, Imaging and Triggered Release," Pharmaceutical Research, Vol. 27, No. 12, 2010, pp. 25692589. doi:10.1007/s11095-010-0233-4

[57] D. S. Bernardi, T. A. Pereira, N. R. Maciel, J. Bortoloto, G. S. Viera, G. C. Oliveira and P. A. Rocha-Filho, "Formation and Stability of Oil-In-Water Nanoemulsions Containing Rice Bran Oil: In Vitro and in Vivo Assessments," Journal of Nanobiotechnology, Vol. 9, 2011, p. 44. doi:10.1186/1477-3155-9-44

[58] F. Shakeel, "Criterion for Excipients Screening in the De- velopment of Nanoemulsion Formulation of Three AntiInflammatory Drugs," Pharmaceutical Development and Technology, Vol. 15, No. 2, 2010, pp. 131-138. doi: $10.3109 / 10837450903055502$

[59] D. I. J. Morrow, P. A. McCarron, A. D. Woolfson and R. F. Donnely, "Innovative Strategies for Enhancing Topical and Transdermal Drug Delivery," The Open Drug Delivery Journal, Vol. 1, 2007, pp. 36-59.

[60] G. M. El Maghraby, B. W. Barry and A. C. Williams, "Liposomes and Skin: From Drug Delivery to Model Membranes," European Journal of Pharmaceutical Sciences, Vol. 34, No. 4-5, 2008, pp. 203-222. doi:10.1016/j.ejps.2008.05.002

[61] M. J. Choi and H. I. Maibach, "Liposomes and Niosomes as Drug Delivery Systems," Skin Pharmacology Physiology, Vol. 18, No. 5, 2005, pp. 209-219. doi:10.1159/000086666

[62] E. Touitou and B. W. Barry, "Enhancement in Drug Delivery," Taylor \& Francis, New York, 1997.

[63] B. Godin and E. Touitoi, "Ethosomes: New Prospects in Trasdermal Delivery," Critical Review in Therapeutic Drug Carrier Systems, Vol. 20, No. 1, 2003, pp. 63-102. doi:10.1615/CritRevTherDrugCarrierSyst.v20.i1.20 